\title{
Digestive ripening: a synthetic method par excellence for core-shell, alloy, and composite nanostructured materials
}

\author{
SRILAKSHMI P BHASKAR and BALAJI R JAGIRDAR* \\ Department of Inorganic and Physical Chemistry, Indian Institute of Science, Bangalore 560 012, India \\ e-mail: jagirdar@ipc.iisc.ernet.in
}

\begin{abstract}
The solvated metal atom dispersion (SMAD) method has been used for the synthesis of colloids of metal nanoparticles. It is a top-down approach involving condensation of metal atoms in low temperature solvent matrices in a SMAD reactor maintained at $77 \mathrm{~K}$. Warming of the matrix results in a slurry of metal atoms that interact with one another to form particles that grow in size. The organic solvent solvates the particles and acts as a weak capping agent to halt/slow down the growth process to a certain extent. This as-prepared colloid consists of metal nanoparticles that are quite polydisperse. In a process termed as digestive ripening, addition of a capping agent to the as-prepared colloid which is polydisperse renders it highly monodisperse either under ambient or thermal conditions. In this, as yet not well-understood process, smaller particles grow and the larger ones diminish in size until the system attains uniformity in size and a dynamic equilibrium is established. Using the SMAD method in combination with digestive ripening process, highly monodisperse metal, core-shell, alloy, and composite nanoparticles have been synthesized. This article is a review of our contributions together with some literature reports on this methodology to realize various nanostructured materials.
\end{abstract}

Keywords. Nanoparticles; core shell; alloy.

\section{Introduction}

In recent years, distinct structures and properties exhibited by materials belonging to the nanometer size regime has stimulated a lot of research interest. The versatile applications of these materials enriched the field of biomedicine, ${ }^{1}$ optoelectronics, ${ }^{2}$ and catalysis ${ }^{3}$ to a great extent due to their unique properties. The property of a nanoparticle strongly depends on its size, shape, interparticle distance and the surrounding environment. ${ }^{4}$ These properties could be tuned precisely by controlling the particle size of the material keeping the other parameters unchanged. The size effect is much more pronounced when the particle size is in the range of $1-10 \mathrm{~nm} .{ }^{5}$ Size dependent property that stems from a polydisperse sample will show an average effect due to different sizes present in the system. In the case of a monodisperse sample, the displayed property could be identified as emerging from a single entity. Hence, tight control over size and size distribution is indispensible for attaining a desired property. In this respect, much attention is focused on developing synthetic strategies

*For correspondence leading to monodispersity and those that are simple to manipulate, easy to scale up and highly reproducible.

We discuss here how digestive ripening, a post synthetic approach is exploited for achieving monodispersity of nanoparticles. An even more remarkable feature of digestive ripening exemplified here is, it could be exercised as a synthetic method towards various heterostructured materials like core-shell particles, nanoalloys, and nanocomposites in combination with the synthetic method, solvated metal atom dispersion (SMAD).

\section{Solvated metal atom dispersion: A top-down approach}

The SMAD set-up for the synthesis of colloidal nanoparticles is comprised of a high vacuum line typically operating at pressures of $10^{-3}$ to $10^{-4}$ torr, a pair of water-cooled copper electrodes with a tungsten crucible (works for most materials) in which the material to be vapourized is placed and a suitable power supply to resistively heat the crucible. The fundamental reactor design was given by Skell, Wescott, Golstein and Engel in $1964{ }^{6}$ The modified SMAD set-up is described in detail elsewhere. ${ }^{7}$ The basic approach of the SMAD 
synthesis involves the growth of atomic/molecular clusters at low temperature starting from the corresponding bulk material. The synthesis involves co-evaporation of the bulk material and stabilizing solvent followed by their co-condensation on the walls of a reactor maintained at $77 \mathrm{~K}$. Warm up of the frozen matrix under argon upon completion of the evaporation results in a slurry of atoms/molecules that interact to form particles that may further grow in size. The solvent can act as a weak capping agent to prevent the growth to some extent. Optionally, an organic ligand/surfactant can be incorporated in the reaction to arrest the growth process and thereby to stabilize the nanoparticles. First report on this approach was from Klabunde and coworkers on the preparation of non-aqueous gold colloid. ${ }^{8}$ From then on, synthesis of a number of metal nanoparticles has been reported by the SMAD method. Apart from metals, the same method has been extended to other systems like metal sulphides, metal halide, etc. ${ }^{9}$ The supremacy of SMAD synthesis over other methodologies is apparent because of its high reproducibility, easy scale up, avoidance of tedious purification processes and high yield. Moreover, this is exceptionally suitable for the synthesis of highly air and moisture sensitive materials which are difficult to prepare by other methods.

\section{The issue of size control: Digestive ripening}

In general, the as-prepared SMAD colloid is quite polydisperse in nature. It can be transformed into a monodisperse sample in a remarkable one-step process termed as digestive ripening which involves refluxing of a polydisperse sample at or near the boiling point of the solvent in the presence of a capping agent and is limited to nanoparticles. In this, not-fully understood process, smaller particles grow in size and larger ones shrink until the system attains uniformity in size. The optimum size obtained for a particular nanoparticle depends on various factors like capping agent, solvent, temperature, etc. The first tentative manifestation of this process was provided by Klabunde and coworkers in $1999 .{ }^{10}$ In this work, $\mathrm{Au}$ nanoparticle surface was ligated by dodecane thiol by heating under reflux which resulted in narrow distribution of size. Subsequent studies on $\mathrm{Au}$ nanoparticles provided more insight into the digestive ripening process. ${ }^{11}$ Apart from the reduction in average particle size and polydispersity, digestive ripening also prompted the formation of 2D and 3D superlattices. In addition to thiols, amines, silanes, and phosphines were also found to be effective in digestive ripening. ${ }^{12}$ It was noted that refluxing of the as-prepared colloid should be done only with digestive ripening agent without surfactants and other impurities. In this context, SMAD provides an excellent starting material for digestive ripening.

Digestive ripening of SMAD prepared colloid was first reported by Stoeva et al. ${ }^{13}$ In this work a highly polydisperse $\mathrm{Au}$-toluene-thiol colloid containing particles of size ranging from 1 to $40 \mathrm{~nm}$ was subjected to digestive ripening. Upon heating under reflux for $1.5 \mathrm{~h}$, transformation to nearly monodisperse colloid with particle size of about 4-4.5 nm took place. Digestive ripening of silver nanoparticles with different ligands also led to monodispersity. ${ }^{14}$ The modisperse particles formed during digestive ripening of SMAD colloid showed large tendency to form extensively ordered superlattices.

Following the same protocol we also made similar kind of observation with copper and zinc. ${ }^{15}$ In the earlier reports on the SMAD synthesis of $\mathrm{Cu}$ nanoparticles, particle agglomeration took place to form spongy ensembles. In our case, the as-prepared $\mathrm{Cu}$ colloid by the SMAD method resulted in broad distribution of well-separated nanoparticles having size in the range of 2.5-8.0 nm. Digestive ripening of this $\mathrm{Cu}$ colloid in the presence of hexadecyl amine (HDA) gave rise to a very narrow size distribution of $2.1 \pm 0.3 \mathrm{~nm}$. Self-assembly into 2D superlattice was also shown by these stabilized $\mathrm{Cu}$ nanoparticles. Similar to this, digestive ripening of the SMAD prepared agglomerated zinc butanone colloid in the presence of HDA resulted in a narrow size distribution of $3.9 \pm 0.3 \mathrm{~nm}$ with well-separated nanoparticles. However, rather strange behaviour was shown by palladium upon digestive ripening. ${ }^{16}$ The as-prepared Pd-butanone-4-tert-butyl toluene-dodecanethiol colloid showed spherical particles of mean size $2.8 \pm 0.1 \mathrm{~nm}$. Even though the colloids were stable against precipitation under Ar, a Pdthiolate complex formation started slowly with time at room temperature. This disintegration of colloid is attributed to the ligand exchange occurring between 2-butanone and dodecanethiol. Digestive ripening of this colloid simply accelerated the thiolate formation. Recently, digestive ripening of indium nanoparticles prepared by the SMAD method under mild condition was reported by Klabunde et al. ${ }^{17}$ Ripening was done in a low boiling solvent methylene chloride at $38^{\circ} \mathrm{C}$. Motivated by the success of the SMAD method followed by the digestive ripening process, this methodology has been extended to binary systems e.g., CdSe also. ${ }^{18}$ In all the above mentioned works, refluxing at the boiling point of solvent is an essential condition for the digestive ripening process. 
Contrary to this, we found that highly monodisperse colloidal $\mathrm{Mg}$ nanoparticles could be obtained by room temperature digestive ripening. ${ }^{19}$ In this work, $\mathrm{Mg}-\mathrm{THF}$ nanoclusters of $25 \mathrm{~nm}$ size undergo a drastic size reduction to $2.8 \pm 0.2 \mathrm{~nm}$ upon mere stirring with HDA ligand. The schematic representation of this process is given in figure 1. The TEM images of the as-prepared $\mathrm{Mg}-\mathrm{THF}$ and the ripened $\mathrm{Mg}-\mathrm{HDA}$ nanoparticles are shown in figure 2.

Pileni et al. reported room temperature ripening of worm-like palladium nanoparticles by stirring with large amount of dodecane thiol. ${ }^{20}$ They showed the transformation of worm-shaped structures to spherical particles revealing shape selectivity of this process. Here, no size reduction is observed since the particle diameter of both the worm-like and the spherical structures remained similar.

In a very recent work, we reported the synthesis of calcium nanoparticles by the SMAD method. ${ }^{21}$ The synthesis of calcium nanoparticles by conventional methods turned out to be a difficult task because of its high reactivity and pyrophoric nature. In our work, the as-prepared, nearly spherical nanoparicles were free standing but quite polydisperse in nature with an average particle size of $8.3 \pm 1.0 \mathrm{~nm}$. As evident from the case of $\mathrm{Mg}$ nanoparticles, here also we observed room temperature ripening by stirring $\mathrm{Ca}$ nanoparticles with HDA taken at bottom of the SMAD reactor. The digestive ripening of the as-prepared Ca nanoparticles in the presence of HDA was apparent from the TEM micrographs showing monodispersity with wellseparated spherical nanoparticles of size $2-3 \mathrm{~nm}$. Based

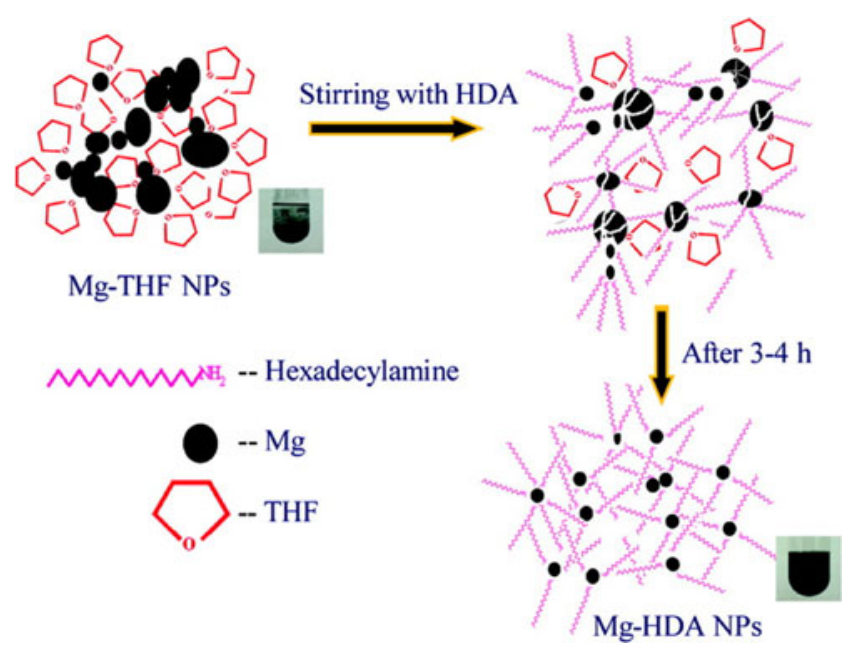

Figure 1. Room temperature digestive ripening of $\mathrm{Mg}-$ THF nanoparticles in the presence of HDA. Reproduced with permission from the American Chemical Society [ref. 19].

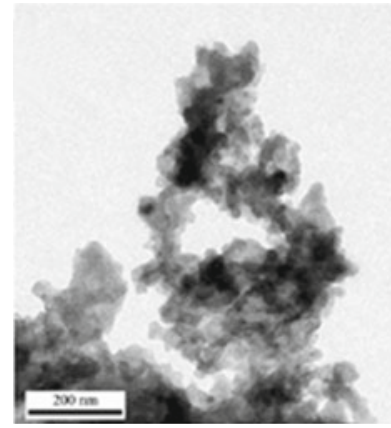

(a)

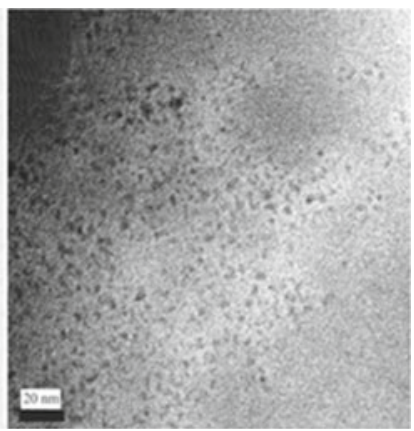

(b)
Figure 2. TEM image of (a) Mg-THF nanoparticles and (b) $\mathrm{Mg}-\mathrm{HDA}$ nanopaticles after room temperature digestive ripening. Reproduced with permission from the American Chemical Society [ref. 19].

on the obtained experimental evidences to date, refluxing need not be a necessary condition atleast in case of certain nanoparticles.

\section{Towards the heterostructures}

Bimetallic nanosystems like core-shell structures, nanocomposites, and nanoalloys have been attracting much attention because of their versatile applications, especially in the field of catalysis. ${ }^{22}$ This is due to the fact that the physicochemical properties of these systems originate from the collective interactions of the constituents and that can be enhanced or extended significantly compared to the individual entity. Numerous methods have been suggested for their synthesis, eventhough most of the methods suffer from certain limitations associated with scale up, reproducibility, monodispersity of particle size and reaction conditions.

Prompted by the advantages of the SMAD method followed by digestive ripening, we explored this combination for realizing bimetallic core-shell structures. The synthetic protocol involves digestive ripening of a mixture of separately prepared as-prepared colloids together with a capping agent at the boiling point of the solvent. Following this protocol, we reported the synthesis of nearly monodisperse $\mathrm{Cu} @ \mathrm{ZnO}$ core-shell structure of average particle size of $3.0 \pm 0.7 \mathrm{~nm}$ by refluxing $\mathrm{Cu}$ and $\mathrm{Zn}$ colloids with HDA in butanone solvent at $80{ }^{\circ} \mathrm{C}$ (figure 3). ${ }^{15}$

Under this mild experimental condition, there was a reduction in the size distribution of both $\mathrm{Cu}$ and $\mathrm{Zn}$ nanoparticles as well as transfer of atoms from one particle to the surface of the other with different elemental composition to form a core-shell structure. The $\mathrm{ZnO}$ shell was formed on the surface of $\mathrm{Cu}-\mathrm{Zn}$ particle due to the preferential oxidation of the $\mathrm{Zn}$ surface. 


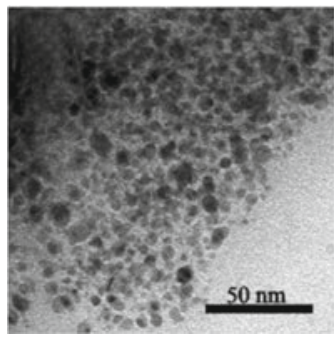

(a)

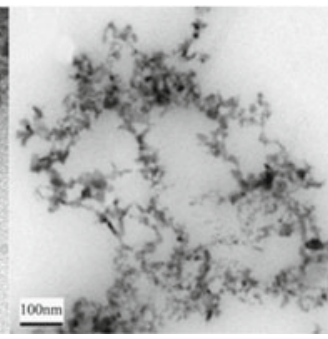

(b)

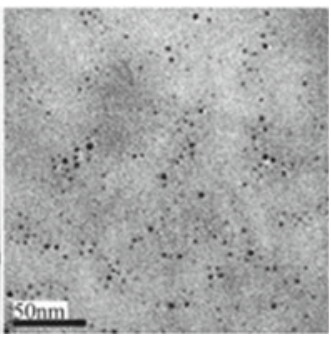

(c)

Figure 3. TEM image of (a) as-prepared $\mathrm{Cu}$-butanone colloid and (b) as-prepared Zn-butanone colloid and (c) $\mathrm{Cu} @ \mathrm{ZnO}$ core-shell nanoparticles. Reproduced with permission from the American Chemical Society [ref. 15].

By the same method, we prepared Au@Pd core-shell nanoparticles which established the reproducibility of this method further. ${ }^{16}$ As mentioned before, rather than leading to monodispersity, refluxing of the Pd-thiol colloid resulted in the rapid formation of $\mathrm{Pd}$-thiolate which was slow at room temperature. The expected competition between formation of thiolate and the core-shell structure is largely suppresed upon mixing $\mathrm{Au}$ and $\mathrm{Pd}$ colloids. Here the core-shell structure formation is initiated even before the mixture is refluxed. Upon refluxing, free $\mathrm{Pd}$ particles bound to thiol get attached to the surface of an Au core hindering the thiolate formation. This core-shell particle was found to be kinetically stable and showed no tendency to form an $\mathrm{Au}-\mathrm{Pd}$ alloy even at higher temperatures. In a similar fashion, $\mathrm{Ag} @ \mathrm{Pd}$ core-shell particles were realized by the digestive ripening process of a mixture of as-prepared dodecanethiol capped Ag and Pd colloids. ${ }^{23}$ During refluxing, polydisperse dodecanethiol-capped Ag nanoparticles transformed into monodisperse $\mathrm{Ag}$ seeds along with small amount of $\mathrm{Ag}$ thiolate. These $\mathrm{Ag}$ seeds favour the breakdown of the Pd clusters into smaller particles which in turn get deposited on the surface of $\mathrm{Ag}$ seeds. This process limited the formation of $\mathrm{Pd}$ thiolate complex.

It is interesting to mention that we also reported the syntheis of $\mathrm{Cu} @ \mathrm{Cu}_{2} \mathrm{O}$ and $\mathrm{Cu}_{2} \mathrm{O}$ (figure 4) nanoparticles using only the SMAD method and studied their catalytic activity in generating hydrogen from ammoniaborane. ${ }^{24}$ The core-shell structure was achieved by the controlled oxidation of SMAD prepared $\mathrm{Cu}$ nanoparticles whereas purging of oxygen for $30 \mathrm{~min}$ resulted in $\mathrm{Cu}_{2} \mathrm{O}$ nanoparticles. The TEM image of $\mathrm{Cu} @ \mathrm{Cu}_{2} \mathrm{O}$ core-shell nanoparticles revealed the formation of more or less monodisperse spherical nanoparticles of average size $7.7 \pm 1.8 \mathrm{~nm}$.

Recently, the synthesis of $\mathrm{Cu} / \mathrm{Mg}$ nanocomposite was realized by the digestive ripening process. ${ }^{25} \mathrm{~A}$ physical mixture of $\mathrm{Mg}$ and $\mathrm{Cu}-\mathrm{HDA}-$ mesitylene colloids was subjected to digestive ripening near the boiling point of mesitylene around $170{ }^{\circ} \mathrm{C}$. The $\mathrm{Cu}$ nanoparticles first underwent narrowing of size distribution with already ripened $\mathrm{Mg}$ nanoparticles, eventually leading to $\mathrm{Cu} / \mathrm{Mg}$ nanocomposite of less than $5 \mathrm{~nm}$ size (figure 5).

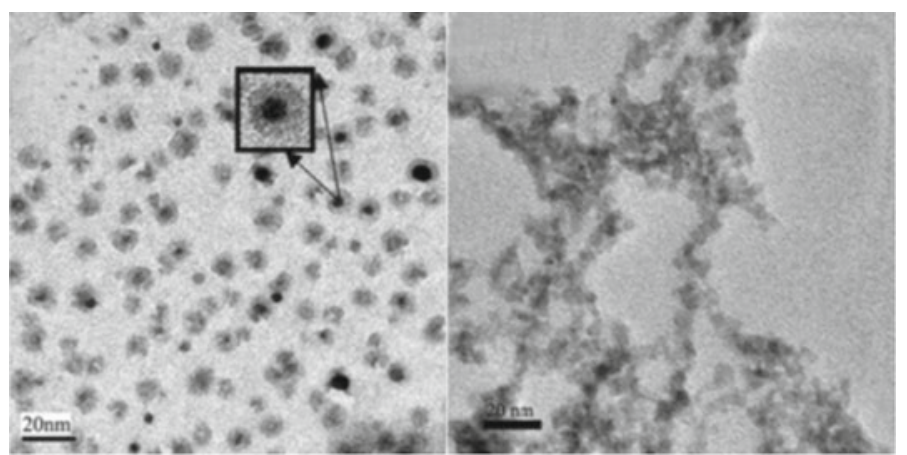

(a)

Figure 4. TEM image of (a) $\mathrm{Cu} @ \mathrm{Cu}_{2} \mathrm{O}$ nanoparticles and (b) $\mathrm{Cu}_{2} \mathrm{O}$ nanoparticles. Reproduced with permission from the Royal Society of Chemistry [ref. 24]. 


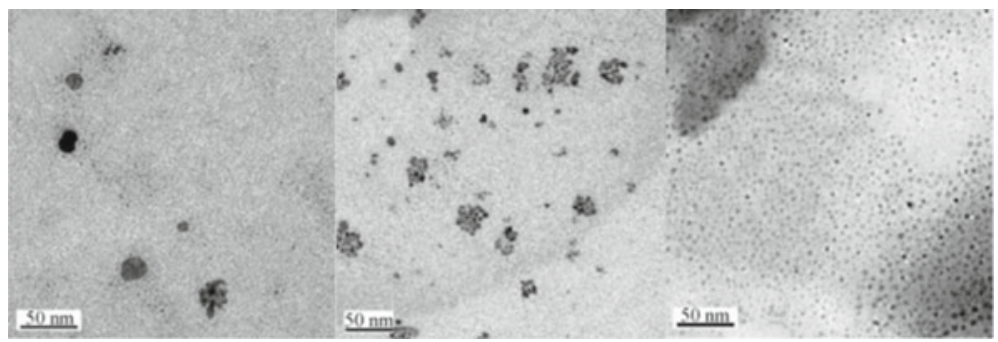

(a)

(b)

(c)

Figure 5. TEM image of (a) physical mixture of $\mathrm{Mg} / \mathrm{Cu}$ nanoparticle before reflux, (b) after $1 \mathrm{~h}$ of reflux and (c) after $6 \mathrm{~h}$ reflux. Reproduced with permission from Wiley-VCH [ref. 25].

Annealing of this $\mathrm{Cu} / \mathrm{Mg}$ nanocomposite resulted in the formation of $\mathrm{Cu} / \mathrm{MgO}$ nanocomposite. The advantage of this procedure is that the difficulty in synthesizing $\mathrm{Cu} / \mathrm{Mg}$ and $\mathrm{Cu} / \mathrm{MgO}$ nanocomposite by conventional method based on the high energy solid state reactions is overcome by simple and mild reaction conditions.

It has been observed in certain cases that by providing sufficient energy dissolution of the core-shell structure leading to formation of an alloy takes place via diffusion of surface atoms of the shell into the bulk of the core. Hodak et al. showed the conversion of $\mathrm{Au} @ \mathrm{Ag}$ core-shell structure into an $\mathrm{Au}-\mathrm{Ag}$ alloy by laser-induced heating. ${ }^{26}$ Under this high energy condition, melting and consequent erosion of the coreshell structure took place to form a homogeneous alloy. Digestive ripening process also has been demonstrated to be effective for nano-alloy formation. Klabunde et al. used this synthetic methodology to prepare alloys of $\mathrm{Cu}$ and $\mathrm{Ag}$ nanoparticles with $\mathrm{Au}$ nanoparticles. ${ }^{27}$ The reaction was carried out in 4-tert-butyl toluene at $198^{\circ} \mathrm{C}$. It was suggested that at this tempetature, interparticle diffusion of atoms from the surface to the centre of nanoparticle brought about the formation of an alloy. Prompted by this, further studies are being carried out in this direction to accomplish different bimetallic nano-alloys.

\section{Conclusions}

Digestive ripening is a well-established process to obtain highly monodisperse nanoparticles. Based on the experimental evidence, we put forward the combination of SMAD and digestive ripening as a powerful and promising technique for realizing core-shell nanoparticles, nanocomposites and nanoalloys. It is also emphasized here that the SMAD method offers propitious solution over the synthetic challenges existing over the synthesis of air sensitive materials. The mechanistic aspects of this coveted process are largely unknown; efforts are in progress in our laboratories to unravel the mechanistic details of the digestive ripening process.

\section{Acknowledgements}

Financial support from the Council of Scientific and Industrial Research (CSIR) is gratefully acknowledged. SPB thanks the CSIR for a fellowship.

\section{References}

1. (a) West J L and Halas N J 2003 Annu. Rev. Biomed. Eng. 5285 (b) Jain P K, Huang X, El-Sayed I H and El-Sayed M A 2008 Acc. Chem. Res. 411578

2. (a) Kruis F E, Fissan H and Peled A 1998 J. Aerosol Sci. 29511 (b) Jiea J, Zhang W, Bello I, Lee C S and Lee S T 2010 Nano Today 5313

3. Polshettiwar V, Luque R, Fihri A, Zhu H, Bouhrara M and Basset J M 2011 Chem. Rev. 1113036

4. Brust M and Kiely C J 2002 Colloids Surf. A: Physicochem. Eng. Asp. 202175

5. Uvarov N F and Boldyrev V V 2001 Russ. Chem. Rev. 70265

6. Skell P S, Wescott L D Jr, Golstein J P and Engel R R 1965 J. Am. Chem. Soc. 872829

7. Klabunde K J, Timms P L, Skell P S and Ittel S 1979 Inorg. Synth. 1959

8. Lin S, Franklin M T and Klabunde K J 1986 Langmuir 2259

9. Heroux D, Ponce A, Cingarapu S and Klabunde KJ 2007 Adv. Funct. Mater. 173562

10. Lin X M, Sorensen C M and Klabunde K J 1999 Chem. Mater. 11198

11. (a) Lin X M, Sorensen C M and Klabunde K J 2000 J. Nanopart. Res. 2157 (b) Prasad B L V, Stoeva S I, Sorensen C M and Klabunde K J 2002 Langmuir 18 7515

12. Prasad B L V, Stoeva S I, Sorensen C M and Klabunde K J 2003 Chem. Mater. 15935

13. Stoeva S I, Klabunde K J, Sorensen C M and Dragieva I 2002 J. Am. Chem. Soc. 1242305

14. Smetana A B, Klabunde K J and Sorensen C M 2005 J. Colloid Interface Sci. 284521 
15. Kalidindi S B and Jagirdar B R 2008 J. Phys. Chem. C 1124042

16. Jose D and Jagirdar B R 2008 J. Phys. Chem. C 112 10089

17. Cingarapu S, Yang Z, Sorensen C M and Klabunde K J 2011 Inorg. Chem. 505000

18. Cingarapu S, Yang Z, Sorensen C M and Klabunde K J 2009 Chem. Mater. 211248

19. Kalidindi S B and Jagirdar B R 2009 Inorg. Chem. 48 4524

20. Naoe K, Petit C and Pileni M P 2007 J. Phys. Chem. C 11116249
21. Sanyal U, Datta R and Jagirdar B R 2012 RSC Adv. 2259

22. Ferrando R, Jellinek J and Johnston R L 2008 Chem. Rev. 108845

23. Jose D and Jagirdar B R 2011 Ind. J. Chem. 50A 1308

24. Kalidindi S B, Sanyal U and Jagirdar B R 2008 Phys. Chem. Chem. Phys. 105870

25. Kalidindi S B and Jagirdar B R 2009 Chem. Asian J. 4 835

26. Hodak $\mathbf{J}$ H, Henglein A, Giersig $M$ and Hartland G V 2000 J. Phys. Chem. B 10411708

27. Smetana A B, Klabunde K J, Sorensen C M, Ponce A A and Mwale B 2006 J. Phys. Chem. B 1102155 